\title{
Study of Three-Component Reaction between Triphenylphosphine, Dimethyl Acetylenedicarboxylate and Thiadiazol Derivatives
}

\author{
FATEME ABOEE-MEHRIZI ${ }^{1}$, MOHAMMAD HOSSEIN MOSSLEMIN ${ }^{2 *}$, \\ ALIREZA HASSANABADI $^{3}$ and MAJID EHSANFAR ${ }^{2}$
}

\author{
${ }^{1}$ Department of Chemistry, Science and Research Branch, Islamic Azad University,Tehran, Iran \\ ${ }^{2}$ Department of Chemistry, Islamic Azad University, Yazd Branch, P.O. Box 89195-155, \\ Yazd, Iran \\ ${ }^{3}$ Department of Chemistry, Islamic Azad University, Zahedan Branch, P.O. Box 98135-978, \\ Zahedan, Iran \\ mhmosslemin@yahoo.com
}

Received 7 May 2012 / Accepted 19 May 2012

\begin{abstract}
Three-component reaction between dimethyl acetylenedicarboxylate (DMAD) and triphenylphosphine in the presence of thiadiazol derivatives leads to the highly fanctionalized, saltfree phosphorus ylides in excellent yields.
\end{abstract}

Keywords: Phosphorus ylides, Dimethyl acetylenedicarboxylate, Triphenylphosphine, Thiadiazol derivatives

\section{Introduction}

Phosphorus ylides are reactive systems, which take part in many reactions of value in organic synthesis $^{1-3}$. Organophosphorus compounds bearing a carbon atom bound directly to a phosphorus atom, are synthetic targets of interest, at least because of their value for a variety of industrial, biological and chemical synthetic uses ${ }^{1-3}$. Several methods have been developed for the preparation of phosphorus ylides. These ylides are usually prepared by treatment of an appropriate phosphonium salt with a base; the corresponding phosphonium salts are usually obtained from the phosphine and an alkyl halide ${ }^{1,2}$. Phosphonium salts are also prepared by Michael addition of phosphorus nucleophiles to activated olefins ${ }^{1,2}$. Reaction of acetylenic esters with triphenylphosphine in the presence of an organic compound possessing an acidichydrogen has been recently reported to produce phosphorus ylides ${ }^{4-6}$. In continuation of our work on the reaction between trivalent phosphorus nucleophiles and acetylenic esters in the presence of organic $\mathrm{NH}, \mathrm{OH}$, or $\mathrm{CH}$-acids ${ }^{7-18}$, we report herein the results of our study on the reaction between dimethyl acetylenedicarboxylate and triphenylphosphine in the presence of thiadiazol derivatives.

\section{Experimental}

Melting points were determined with an Electrothermal 9100 apparatus. Elemental analyses were performed using a Costech ECS 4010 CHNS-O analyser. Mass spectra were recorded on a Finnigan-Mat 8430 mass spectrometer operating at an ionisation potential of $70 \mathrm{eV}$. 
IR spectra were recorded on a Shimadzu IR-470 spectrometer. ${ }^{1} \mathrm{H},{ }^{13} \mathrm{C}$ and ${ }^{31} \mathrm{P}$ NMR spectra were recorded on Bruker DRX-500 Avance spectrometer in $\mathrm{d}_{6}$-DMSO using TMS as internal standard or $85 \% \mathrm{H}_{3} \mathrm{PO}_{4}$ as external standard. The chemicals used in this work were purchased from Fluka (Buchs, Switzerland) and were used without further purification.

\section{General procedure for preparation of compounds $\mathbf{5 a - c}$ and $\mathbf{6 a - c}$}

To a magnetically stirred solution of triphenylphosphine $(1 \mathrm{mmol})$ and thiadiazol derivatives $(1 \mathrm{mmol})$ in acetone $(15 \mathrm{~mL})$ was added dropwise a mixture of dimethyl acetylenedicarboxylate $(1 \mathrm{mmol})$ in acetone $(2 \mathrm{~mL})$ at room temperature over $2 \mathrm{~min}$. The reaction mixture was then stirred for $24 \mathrm{~h}$ at room temperature. The organic layer was then concentrated and passed trough a silica gel pad eluting by hexane-ethyl acetate (2:1) mixture. Solvent was evaporated and the product was obtained as a powder yellow.

Dimethyl 3-(triphenyl- $\lambda^{5}$-phosphanylidene)-2-(5-(5nitrothiophen-2-yl-)1,3,4-thiadiazol2-yl-amino)succinate (5a)

Yield: 93\%; Yellow powder; m.p. 168-169 ${ }^{\circ} \mathrm{C}$. IR $(\mathrm{KBr})\left(v_{\max }, \mathrm{cm}^{-1}\right)$ : 1731, $1695(\mathrm{C}=\mathrm{O})$. Calcd. for $\mathrm{C}_{30} \mathrm{H}_{25} \mathrm{~N}_{4} \mathrm{O}_{6} \mathrm{PS}_{2}$ : C, 56.95; H, 3.98; N, 8.86, \%. Found: C, 56.77; H, 3.69; N, 9.11\%. MS $(\mathrm{m} / \mathrm{z}, \%): 632\left(\mathrm{M}^{+}, 7\right) .{ }^{1} \mathrm{H}$ NMR (500 MHz, $\left.\mathrm{d}_{6}-\mathrm{DMSO}\right): \delta 3.61\left(3 \mathrm{H}, \mathrm{s}, \mathrm{OCH}_{3}\right), 3.68(3 \mathrm{H}, \mathrm{s}$, $\left.\mathrm{OCH}_{3}\right), 4.45\left(1 \mathrm{H}, \mathrm{dd},{ }^{3} J_{\mathrm{HP}}=5 \mathrm{~Hz},{ }^{3} J_{\mathrm{HH}}=10 \mathrm{~Hz}, \mathrm{P}-\mathrm{C}-\mathrm{CH}\right), 6.71\left(1 \mathrm{H}, \mathrm{d},{ }^{3} J_{\mathrm{HH}}=10 \mathrm{~Hz}, \mathrm{NH}\right)$, 6.81-7.49 (17 H, m, aromatic). ${ }^{13} \mathrm{C}$ NMR (125.8 MHz, $\left.\mathrm{d}_{6}-\mathrm{DMSO}\right): \delta 48.4\left(\mathrm{~d},{ }^{1} J_{\mathrm{PC}}=122 \mathrm{H}_{\mathrm{Z}}\right.$, $\mathrm{C}=\mathrm{P})$, 52.6, 53.9, $\left(2 \mathrm{OCH}_{3}\right), 60.7\left(\mathrm{~d},{ }^{2} J_{\mathrm{PC}}=15 \mathrm{~Hz}, \mathrm{CH}\right), 126.9\left(\mathrm{~d},{ }^{1} J_{\mathrm{PC}}=91 \mathrm{~Hz}\right), 129.7\left({ }^{2} J_{\mathrm{PC}}=\right.$ $12 \mathrm{~Hz}), 132.2\left(\mathrm{~d},{ }^{4} J_{\mathrm{PC}}=2 \mathrm{H}_{\mathrm{Z}}\right), 133.7\left(\mathrm{~d},{ }^{3} J_{\mathrm{PC}}=10 \mathrm{~Hz}\right), 120.8,122.8,128.0,128.4,128.7,128.8$, 128.9, 131.6, 131.7, 135.2, 136.4, 137.3, 140.1, 144.5 (14C, aromatic), 167.3 (d, ${ }^{2} J_{\mathrm{PC}}=12 \mathrm{~Hz}$ $\mathrm{C}=\mathrm{O}), 171.4\left(\mathrm{~d},{ }^{3} J_{\mathrm{PC}}=17 \mathrm{~Hz} \mathrm{C}=\mathrm{O}\right) .{ }^{31} \mathrm{P}$ NMR (202.5 MHz, $\left.\mathrm{d}_{6}-\mathrm{DMSO}\right): \delta 23.58$.

Dimethyl 3-(triphenyl- $\lambda^{5}$-phosphanylidene)-2-(5-(5nitrofuran-2-yl-)1,3,4-thiadiazol2-yl-amino)succinate (5b)

Yield: 91\%; Yellow powder; m.p. 193-194 ${ }^{\circ} \mathrm{C}$. IR (KBr) $\left(v_{\max }, \mathrm{cm}^{-1}\right)$ : 1731,1690 (C=O). Calcd. for $\mathrm{C}_{30} \mathrm{H}_{25} \mathrm{~N}_{4} \mathrm{O}_{7} \mathrm{PS}$ : C, 58.44; H, 4.09; N, 9.09, \%. Found: C, 58.66; H, 4.16; N, 8.97, \%. MS $(\mathrm{m} / \mathrm{z}, \%): 616\left(\mathrm{M}^{+}, 7\right) .{ }^{1} \mathrm{H}$ NMR (500 MHz, d $\left.\mathrm{d}_{6}-\mathrm{DMSO}\right): \delta 3.53\left(3 \mathrm{H}, \mathrm{s}, \mathrm{OCH}_{3}\right), 3.60(3 \mathrm{H}, \mathrm{s}$, $\left.\mathrm{OCH}_{3}\right), 4.51\left(1 \mathrm{H}, \mathrm{dd},{ }^{3} J_{\mathrm{HP}}=5 \mathrm{~Hz},{ }^{3} J_{\mathrm{HH}}=10 \mathrm{~Hz}, \mathrm{P}-\mathrm{C}-\mathrm{CH}\right), 6.93\left(1 \mathrm{H}, \mathrm{d},{ }^{3} \mathrm{~J}_{\mathrm{HH}}=10 \mathrm{~Hz}, \mathrm{NH}\right)$, 6.95-7.89 (17 H, m, aromatic). ${ }^{13} \mathrm{C}$ NMR (125.8 MHz, d $\mathrm{d}_{6}$-DMSO): $\delta 46.4\left(\mathrm{~d},{ }^{1} J_{\mathrm{PC}}=121 \mathrm{~Hz}\right.$, $\mathrm{C}=\mathrm{P}), 52.3$, $53.4\left(2 \mathrm{OCH}_{3}\right), 61.7\left(\mathrm{~d},{ }^{2} J_{\mathrm{PC}}=15 \mathrm{~Hz}, \mathrm{CH}\right), 127.9\left(\mathrm{~d},{ }^{1} J_{\mathrm{PC}}=91 \mathrm{~Hz}\right), 128.7\left({ }^{2} J_{\mathrm{PC}}=\right.$ $12 \mathrm{~Hz}), 131.2\left(\mathrm{~d},{ }^{4} J_{\mathrm{PC}}=2 \mathrm{~Hz}\right), 132.7\left(\mathrm{~d},{ }^{3} J_{\mathrm{PC}}=10 \mathrm{~Hz}\right), 121.8,123.16,124.8,129.0,129.4,130.1$, 130.7, 130.8, 131.3, 131.6, 137.7, 139.4, 140.2, 142.6 (14C, aromatic), 166.2 (d, ${ }^{2} J_{\mathrm{PC}}=12 \mathrm{~Hz}$ $\mathrm{C}=\mathrm{O}), 170.4\left(\mathrm{~d},{ }^{3} \mathrm{~J}_{\mathrm{PC}}=17 \mathrm{~Hz} \mathrm{C}=\mathrm{O}\right) .{ }^{31} \mathrm{P}$ NMR (202.5 MHz, $\left.\mathrm{d}_{6}-\mathrm{DMSO}\right): \delta 24.87$.

Dimethyl 3-(triphenyl- $\lambda^{5}$-phosphanylidene)-2-(5-(1-methyl-5-nitro-1H-imidazol-2yl)-1,3,4-thiadiazol-2-yl-amino)succinate (5c)

Yield: 87\%; Yellow powder; m.p. 196-197 ${ }^{\circ} \mathrm{C}$. IR (KBr) $\left(v_{\max }, \mathrm{cm}^{-1}\right)$ : 1734, $1698(\mathrm{C}=\mathrm{O})$. Calcd. for $\mathrm{C}_{30} \mathrm{H}_{27} \mathrm{~N}_{6} \mathrm{O}_{6} \mathrm{PS}$ : C, 57.14; H, 4.32; N, 13.33 \%. Found: C, 56.98; H, 4.40; N, 13.19\%. MS $(\mathrm{m} / \mathrm{z}, \%): 643\left(\mathrm{M}^{+}, 5\right) .{ }^{1} \mathrm{H}$ NMR (500 MHz, d $\left.\mathrm{d}_{6}-\mathrm{DMSO}\right): \delta 3.48\left(3 \mathrm{H}, \mathrm{s}, \mathrm{CH}_{3}\right), 3.61(3 \mathrm{H}, \mathrm{s}$, $\left.\mathrm{OCH}_{3}\right), 3.68\left(3 \mathrm{H}, \mathrm{s}, \mathrm{OCH}_{3}\right), 4.61\left(1 \mathrm{H}, \mathrm{dd},{ }^{3} \mathrm{~J}_{\mathrm{HP}}=5 \mathrm{~Hz},{ }^{3} J_{\mathrm{HH}}=10 \mathrm{~Hz}, \mathrm{P}-\mathrm{C}-\mathrm{CH}\right), 7.01(1 \mathrm{H}, \mathrm{d}$, $\left.{ }^{3} J_{\mathrm{HH}}=10 \mathrm{~Hz}, \mathrm{NH}\right), 7.27-8.01$ (16 H, m, aromatic). ${ }^{13} \mathrm{CNMR}$ (125.8 MHz, $\mathrm{d}_{6}$-DMSO): $\delta 20.9$ (CH3), 47.5 (2CH2), 48.7 (2 CH2), $48.9\left(\mathrm{~d},{ }^{1} J_{\mathrm{PC}}=122 \mathrm{~Hz}, \mathrm{C}=\mathrm{P}\right), 52.6,53.9\left(2 \mathrm{OCH}_{3}\right), 60.7$ $\left(\mathrm{d},{ }^{2} J_{\mathrm{PC}}=15 \mathrm{~Hz}, \mathrm{CH}\right), 126.9\left(\mathrm{~d},{ }^{1} J_{\mathrm{PC}}=91 \mathrm{~Hz}\right), 129.7\left({ }^{2} J_{\mathrm{PC}}=12 \mathrm{~Hz}\right), 132.2\left(\mathrm{~d},{ }^{4} J_{\mathrm{PC}}=2 \mathrm{~Hz}\right), 133.7$ (d, $\left.{ }^{3} J_{\mathrm{PC}}=10 \mathrm{~Hz}\right), 120.8,122.8,128.0,128.4,128.7,128.8,128.9,131.6,131.7,144.5,144.8,146.6$, 147.3, (13C, aromatic), $167.3\left(\mathrm{~d},{ }^{2} J_{\mathrm{PC}}=12 \mathrm{~Hz} \mathrm{C}=\mathrm{O}\right), 171.4$ (d, $\left.{ }^{3} J_{\mathrm{PC}}=17 \mathrm{~Hz} \mathrm{C}=\mathrm{O}\right) .{ }^{31} \mathrm{P} \mathrm{NMR}$ (202.5 MHz, $\mathrm{d}_{6}$-DMSO): $\delta$ 23.37. 
Dimethyl 2-(triphenyl- $\lambda^{5}$-phosphanylidene)-3-(4-(5-(5nitrothiophen-2-yl-)1,3,4thiadiazol-2-yl-piperazin-1-yl)succinate (6a)

Yield: 90\%; Yellow powder; m.p. 199-200 ${ }^{\circ} \mathrm{C}$. IR (KBr) $\left(v_{\max }, \mathrm{cm}^{-1}\right): 1731,1690(\mathrm{C}=\mathrm{O})$. Calcd. for $\mathrm{C}_{34} \mathrm{H}_{32} \mathrm{~N}_{5} \mathrm{O}_{6} \mathrm{PS}_{2}$ : C, 58.19; H, 4.60; N, 9.98, \%. Found: C, 58.36; H, 4.76; N, 9.69, \%. MS (m/z, \%): $701\left(\mathrm{M}^{+}, 1\right) .{ }^{1} \mathrm{H}$ NMR (500 MHz, $\left.\mathrm{d}_{6}-\mathrm{DMSO}\right): \delta 2.67\left(4 \mathrm{H}, \mathrm{t}, 2 \mathrm{CH}_{2}\right)$, $3.16\left(4 \mathrm{H}, \mathrm{t}, 2 \mathrm{CH}_{2}\right), 3.61\left(3 \mathrm{H}, \mathrm{s}, \mathrm{OCH}_{3}\right), 3.68\left(3 \mathrm{H}, \mathrm{s}, \mathrm{OCH}_{3}\right), 4.87\left(1 \mathrm{H}, \mathrm{dd},{ }^{3} J_{\mathrm{HP}}=5 \mathrm{H}_{\mathrm{Z}}\right.$, P-C-CH), 6.82-7.59 (17 H, m, aromatic). ${ }^{13} \mathrm{C}$ NMR (125.8 MHz, d 6 -DMSO): $\delta 46.6$ $\left(2 \mathrm{CH}_{2}\right), 47.3\left(2 \mathrm{CH}_{2}\right), 48.4\left(\mathrm{~d},{ }^{1} J_{\mathrm{PC}}=122 \mathrm{~Hz} \mathrm{C}=\mathrm{P}\right), 52.6,53.9\left(2 \mathrm{OCH}_{3}\right), 60.7\left(\mathrm{~d},{ }^{2} J_{\mathrm{PC}}=15\right.$ $\mathrm{Hz}, \mathrm{CH}), 126.9\left(\mathrm{~d},{ }^{1} J_{\mathrm{PC}}=91 \mathrm{~Hz}\right), 129.7\left({ }^{2} J_{\mathrm{PC}}=12 \mathrm{~Hz}\right), 132.2\left(\mathrm{~d},{ }^{4} J_{\mathrm{PC}}=2 \mathrm{~Hz}\right), 133.7$ $\left(\mathrm{d},{ }^{3} J_{\mathrm{PC}}=10 \mathrm{~Hz}\right), 120.8,122.8,128.0,128.4,128.7,128.8,128.9,131.6,131.7,135.2$, 136.4, 137.3, 140.1, 144.5 (14 C, aromatic), $167.3\left(\mathrm{~d},{ }^{2} J_{\mathrm{PC}}=12 \mathrm{~Hz} \mathrm{C}=\mathrm{O}\right), 171.4\left(\mathrm{~d},{ }^{3} J_{\mathrm{PC}}=\right.$ $\left.17 \mathrm{H}_{\mathrm{Z}} \mathrm{C}=\mathrm{O}\right) .{ }^{31} \mathrm{P}$ NMR (202.5 MHz, $\mathrm{d}_{6}$-DMSO): $\delta 23.56$.

Dimethyl 2-(triphenyl- $\lambda^{5}$-phosphanylidene)-3-(4-(5-(5nitrofuran-2-yl-)1,3,4-thiadiazol2-yl-piperazin-1-yl)succinate (6b)

Yield: 88\%; Yellow powder; m.p. 201-202 ${ }^{\circ} \mathrm{C}$. IR (KBr) $\left(v_{\max }, \mathrm{cm}^{-1}\right)$ : 1735, $1701(\mathrm{C}=\mathrm{O})$. Calcd. for $\mathrm{C}_{34} \mathrm{H}_{32} \mathrm{~N}_{5} \mathrm{O}_{7} \mathrm{PS}$ : C, 59.56; H, 4.70; N, 10.21, \%. Found: C, 59.74; H, 4.81; N, 10.53\%. MS (m/z, \%): $685\left(\mathrm{M}^{+}, 3\right) .{ }^{1} \mathrm{H}$ NMR (500 MHz, d $\left.{ }_{6}-\mathrm{DMSO}\right): \delta 2.66\left(4 \mathrm{H}, \mathrm{t}, 2 \mathrm{CH}_{2}\right), 3.18\left(4 \mathrm{H}, \mathrm{t}, 2 \mathrm{CH}_{2}\right), 3.61(3 \mathrm{H}$, s, $\left.\mathrm{OCH}_{3}\right), 3.68\left(3 \mathrm{H}, \mathrm{s}, \mathrm{OCH}_{3}\right), 4.45\left(1 \mathrm{H}, \mathrm{dd},{ }^{3} J_{\mathrm{HP}}=5 \mathrm{H}_{\mathrm{Z}}, \mathrm{P}-\mathrm{C}-\mathrm{CH}\right), 6.81-7.49(17 \mathrm{H}, \mathrm{m}$, aromatic). ${ }^{13} \mathrm{C}$ NMR (125.8 MHz, d $\mathrm{d}_{6}$-DMSO): $\delta 47.6\left(2 \mathrm{CH}_{2}\right), 47.9\left(2 \mathrm{CH}_{2}\right), 49.4\left(\mathrm{~d},{ }^{1} J_{\mathrm{PC}}=122 \mathrm{~Hz}\right.$, $\mathrm{C}=\mathrm{P}), 52.7,54.1\left(2 \mathrm{OCH}_{3}\right), 60.1\left(\mathrm{~d},{ }^{2} J_{\mathrm{PC}}=15 \mathrm{~Hz}, \mathrm{CH}\right), 121.4\left(\mathrm{~d},{ }^{1} J_{\mathrm{PC}}=91 \mathrm{~Hz}\right), 128.7\left({ }^{2} J_{\mathrm{PC}}=12\right.$ $\mathrm{Hz}), 132.6\left(\mathrm{~d},{ }^{4} J_{\mathrm{PC}}=2 \mathrm{~Hz}\right), 133.6\left(\mathrm{~d},{ }^{3} J_{\mathrm{PC}}=10 \mathrm{~Hz}\right), 121.8,124.3,127.0,127.4,128.9,129.6,129.9$, 131.5, 131.9, 134.4, 136.7, 137.3,140.3,144.8 (14 C, aromatic), 168.2 (d, ${ }^{2} J_{\mathrm{PC}}=12 \mathrm{~Hz} \mathrm{C}=\mathrm{O}$ ), 170.4 $\left(\mathrm{d},{ }^{3} \mathrm{~J}_{\mathrm{PC}}=17 \mathrm{~Hz} \mathrm{C}=\mathrm{O}\right) .{ }^{31} \mathrm{P}$ NMR $\left(202.5 \mathrm{MHz}, \mathrm{d}_{6}\right.$-DMSO): $\delta 23.71$.

Dimethyl 3-(triphenyl- $\lambda^{5}$-phosphanylidene)-2-(5-(1-methyl-5-nitro-1H-imidazol-2yl)-1,3,4-thiadiazol-2-yl-piperazin-1-yl)succinate (6c)

Yield: 85\%; Yellow powder; m.p. 207-208 ${ }^{\circ} \mathrm{C}$. IR $(\mathrm{KBr})\left(v_{\max }, \mathrm{cm}^{-1}\right)$ : 1738, $1695(\mathrm{C}=\mathrm{O})$. Calcd. for $\mathrm{C}_{34} \mathrm{H}_{34} \mathrm{~N}_{7} \mathrm{O}_{6} \mathrm{PS}$ : C, 58.36; H, 4.90; N, 14.01\%. Found: C, 58.98; H, 4.52; N, 13.99\%. MS ( $\left.\mathrm{m} / \mathrm{z}, \%\right)$ : $699\left(\mathrm{M}^{+}, 1\right) .{ }^{1} \mathrm{H}$ NMR (500 MHz, d $\left.{ }_{6}-\mathrm{DMSO}\right): \delta 2.56\left(4 \mathrm{H}, \mathrm{t}, 2 \mathrm{CH}_{2}\right), 3.12\left(4 \mathrm{H}, \mathrm{t}, 2 \mathrm{CH}_{2}\right), 3.59(3 \mathrm{H}$, s, $\left.\mathrm{CH}_{3}\right), 3.61\left(3 \mathrm{H}, \mathrm{s}, \mathrm{OCH}_{3}\right), 3.68\left(3 \mathrm{H}, \mathrm{s}, \mathrm{OCH}_{3}\right), 4.21\left(1 \mathrm{H}, \mathrm{dd},{ }^{3} J_{\mathrm{HP}}=5 \mathrm{~Hz}, \mathrm{P}-\mathrm{C}-\mathrm{CH}\right), 7.17-8.02$ (16 H, m, aromatic). ${ }^{13} \mathrm{C}$ NMR (125.8 MHz, d $\mathrm{d}_{6}$-DMSO): $\delta 20.9$ (CH3), 49.5 (2 CH2), 49.7 (2 CH2), $50.4\left(\mathrm{~d},{ }^{1} J_{\mathrm{PC}}=122 \mathrm{~Hz}, \mathrm{C}=\mathrm{P}\right), 51.6,53.7\left(2 \mathrm{OCH}_{3}\right), 60.7\left(\mathrm{~d},{ }^{2} J_{\mathrm{PC}}=15 \mathrm{~Hz}, \mathrm{CH}\right), 127.9$ $\left(\mathrm{d},{ }^{1} J_{\mathrm{PC}}=91 \mathrm{~Hz}\right), 130.7\left({ }^{2} J_{\mathrm{PC}}=12 \mathrm{~Hz}\right), 131.2\left(\mathrm{~d},{ }^{4} J_{\mathrm{PC}}=2 \mathrm{~Hz}\right), 133.6\left(\mathrm{~d},{ }^{3} J_{\mathrm{PC}}=10 \mathrm{~Hz}\right), 122.2,122.9$, 128.0, 129.4, 129.7, 130.8, 131.9, 132.6, 135.7, 136.2,138.9,141.5, 144.5 (13 C, aromatic), 167.5 $\left(\mathrm{d},{ }^{2} J_{\mathrm{PC}}=12 \mathrm{~Hz} \mathrm{C}=\mathrm{O}\right), 172.4\left(\mathrm{~d},{ }^{3} J_{\mathrm{PC}}=17 \mathrm{~Hz} \mathrm{C}=\mathrm{O}\right) .{ }^{31} \mathrm{P}$ NMR $\left(202.5 \mathrm{MHz}, \mathrm{d}_{6}-\mathrm{DMSO}\right): \delta 24.15$.

\section{Results and Discussion}

The reaction of the [5-aryl-(1,3,4-thiadiazol-2-amine)] $\mathbf{1}$ or [5-aryl-(1,3,4-thiadiazol-2-ylpiperazine)] 2 with dimethyl acetylenedicarboxylate (DMAD) 3 in the presence of triphenylphosphine $\mathbf{4}$ leads to the corresponding ylides $\mathbf{5}$ and $\mathbf{6}$ in good yields

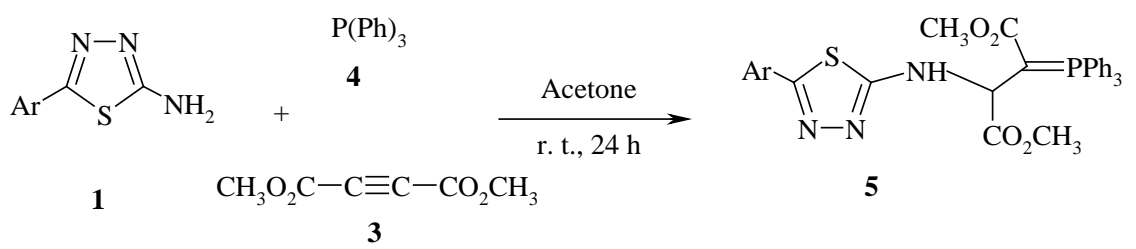




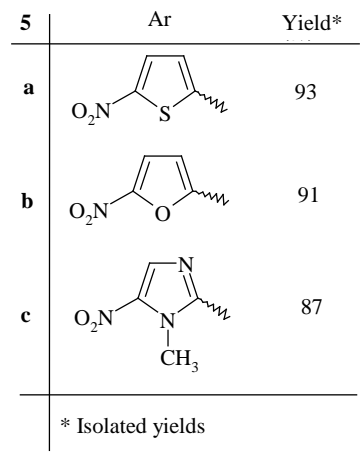

Figure 1. Condensation of dimethyl acetylenedicarboxylate and [5-aryl-(1,3,4-thiadiazol-2amine)] in the presence of triphenylphosphine

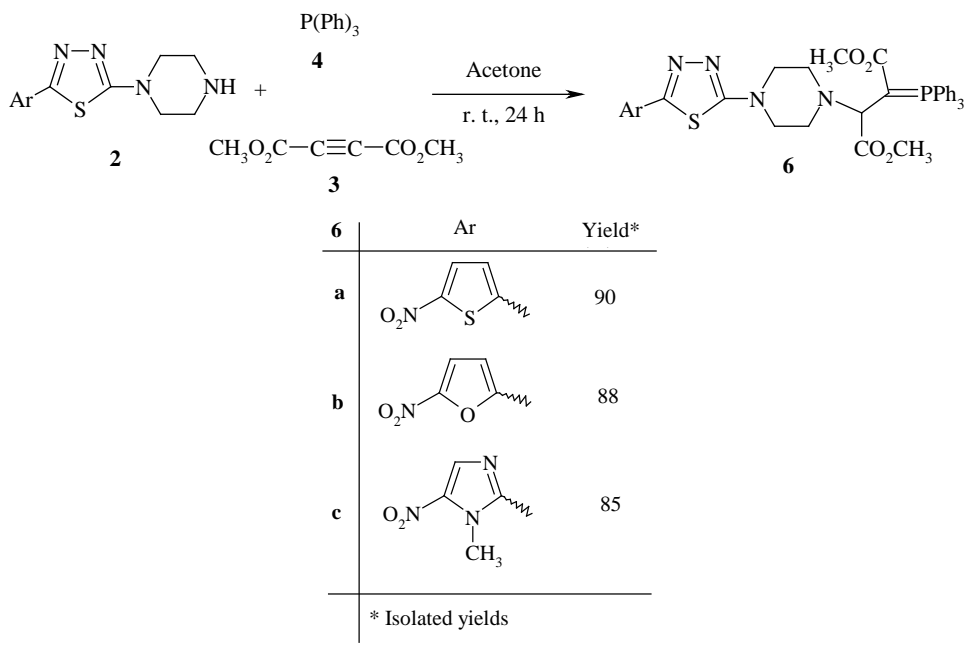

Figure 2. Condensation of dimethyl acetylenedicarboxylate and [5-aryl-(1,3,4-thiadiazol-2yl-piperazine)] in the presence of triphenylphosphine

The products 5a-c and 6a-c were all new compounds. Their structures were deduced from their elemental analyses and spectral data. The ${ }^{1} \mathrm{H}$ NMR spectrum of $\mathbf{5 a}$ displays two sharp lines $(\delta 3.61,3.68 \mathrm{ppm})$ for the methoxy groups and two doublet at $4.45\left({ }^{3} \mathrm{~J}_{\mathrm{PH}}=5 \mathrm{H}_{\mathrm{Z}}\right.$, $\left.{ }^{3} J_{\mathrm{HH}}=10 \mathrm{H}_{\mathrm{Z}}, \mathrm{P}=\mathrm{C}-\mathrm{CH}\right)$ for methine proton which is coupled with phosphorus atom and $\mathrm{NH}$, a doublet signal at $\delta=6.71$ is related to proton of $\mathrm{N}-\mathrm{H}$ proton and multiplets signal between 6.81-7.49 (17 H, m, aromatic) for aromatic protons. The ${ }^{31} \mathrm{P}$ NMR spectrum of compound $\mathbf{5 a}$ consists of one signal at 23.58. This shift is similar to those observed for other stable phosphorus ylides ${ }^{19,20}$. The structural assignments made on the basis of the NMR spectra of compounds 5a-c and 6a-c are supported by their IR spectra. The IR spectrum showed an absorption bond at $3365 \mathrm{~cm}^{-1}$ for NH group. The carbonyl stretching vibration observed as strong absorption bonds at 1695 and $1731 \mathrm{~cm}^{-1}$ for the ester groups.

It is reasonable to assume that ylide $\mathbf{5}$ or $\mathbf{6}$ results from the initial addition of triphenylphosphine to DMAD and subsequent protonation of the 1:1 adduct by the NHacidic thiadiazol derivatives. The positively charged ion $\mathbf{7}$ is then attacked by anion $\mathbf{8}$ to form the phosphorane 5 (Figure 3). 


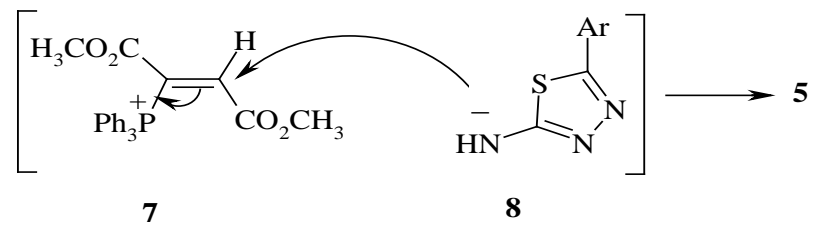

Figure 3. Suggested mechanism for formation of compound 5 or $\mathbf{6}$

\section{Conclusion}

In summary simple, one-pot and three-component reaction between dimethyl acetylenedicarboxylates (DMAD) and triphenylphosphine in the presence of thiadiazol derivatives leads to highly fanctionalized, salt-free phosphorus ylides in excellent yields. The present method carries the advantage that the reaction is performed under neutral conditions and starting materials can be mixed without any activation or modification.

\section{References}

1. Engel R, Synthesis of Carbon-Phosphorus Bonds (CRC Press, Boca Raton, FL, 1988.

2. Corbridge D E C, Phosphorus, An Outline of Chemistr, Biochemistry and Uses ( $5^{\text {th }}$ Edn., Elsevier, Amsterdam, 1995).

3. Cadogan J I G, Organophosphorus Reagents in Organic Synthesis (Academic Press, New York, 1979).

4. Yavari I and Karimi E, Phosphorus, Sulfur and Silicon Relat Elem., 2007, 595.

5. Islami M R, Amrollahi M A and Iranmanesh M, Arkivoc, 2009, 35.

6. Alizadeh A, Zohreh N and Zhu L G, Synthesis, 2009, 3, 464.

7. Hassanabadi A, Hosseini-Tabatabaei M R, Shahraki M and Anary-Abbasinejad M, Ghoroghchian S, J Chem Res., 2011,11.

8. Yavari I, Anary-Abbasinejad M and Hossaini Z, Org Biomol Chem., 2003, 3, 560.

9. Anary-abbasinejad M and Ascarrian N, J Chem Res., 2007, 11.

10. Anary-Abbasinejad M, Rostami N, Parhami A and Hassanabadi A, J Chem Res., 2007, 257.

11. Anary-Abbasinejad M, Hassanabadi A and Anaraki-Ardakani H, J Chem Res., 2007, 455.

12. Anary-Abbasinejad M and Hassanabadi A, J Chem Res., 2007, 475.

13. Anary-Abbasinejad M, Anaraki-Ardakani H, Dehghan A, Hassanabadi A and Seyedmir M R, J Chem Res., 2007, 574.

14. Anary-Abbasinejad M, Hassanabadi A and Mazraeh-Seffid M, J Chem Res., 2007, 708.

15. Anary-Abbasinejad M, Charkhati K and Hassanabadi A, J Chem Res., 2009, 319.

16. Mosslemin M H, Anary-Abbasinejad M, Hassanabadi A and Bagheri M A, J Sulfur Chem., 2010, 31, 135.

17. Hassanabadi A, Mosslemin M H, Anary-Abbasinejad M and Khalesi H, Synth Commun., 2011, 41, 1529.

18. Mohebat R, Iravani Moghaddam M, Askari Motlagh R, Hassanabadi A, KalamiYazdi M, J Chem Res., 2011, 564.

19. Tebby J C, In Phosphorus-31 NMR Spectroscopy in Stereochemical Analysis, Verkade J C and Quin L D(Eds.); VCH: Weinheim, 1987; chap. 1, 1-60.

20. Vedejs E and Snoble K A J, J Am Chem Soc., 1973, 95, 5778. 